\title{
A Case of Cerebrospinal Fluid Hypovolemia With Orthostatic Dysregulation
}

\author{
Yuko Ohashi ${ }^{\text {a }}$, Tatsuo Fuchigami ${ }^{\text {a, c }}$, Satoko Takahashia, Yuko Moriuchi ${ }^{\mathrm{a}}$, \\ Tetsuharu Kamioka ${ }^{a}$, Koichi Takahashi ${ }^{b}$
}

\begin{abstract}
We present the case of a previously healthy 13-year-old boy with cerebrospinal fluid (CSF) hypovolemia followed by orthostatic dysregulation (OD). OD is a clinical entity that presents with various symptoms, such as vertigo when standing up, syncope during standing, and palpitation. The essential mechanism of CSF hypovolemia is thought to be that CSF leakage at the spine level decreases the volume and pressure of the CSF. The causes of CSF hypovolemia in children remain unclear, and patients with this condition complain of symptoms, such as orthostatic headache, vertigo, and tinnitus; therefore, it is represented by OD. Our patient's symptoms began with an orthostatic headache. Consequently, the patient was diagnosed with OD using the orthostatic test. He was treated with midodrine, but his symptoms did not improve. Thereafter, he was diagnosed with CSF hypovolemia using radioisotope cisternography and computed tomography myelography. Epidural blood patch treatment was performed, after which his symptoms improved, and he was able to return to school life. Some patients with CSF hypovolemia are often misdiagnosed with OD, autonomic nervous system dysfunction, or psychiatric disorders. Therefore, it is important to note that CSF hypovolemia is not rare in children and that it may be found among children with OD, chronic daily headaches, and indeterminate complaints, which interfere with school and daily activities.
\end{abstract}

Keywords: Cerebrospinal fluid hypovolemia; Orthostatic dysregulation; Postural orthostatic tachycardia syndrome; Orthostatic headache; Radioisotope cisternography; Computed tomography myelography; Epidural blood patch

\section{Introduction}

School-aged children and adolescents are susceptible to ortho-

Manuscript submitted September 21, 2021, accepted November 22, 2021 Published online November 29, 2021

aDepartment of Pediatrics, IMS Fujimi General Hospital, Saitama, Japan bepartment of Neurosurgery, Sanno Hospital, Tokyo, Japan

${ }^{\mathrm{c} C}$ Corresponding Author: Tatsuo Fuchigami, Department of Pediatrics, IMS Fujimi General Hospital, 1967-1, Tsuruma, Fujimi-shi, Saitama 354-0021, Japan. Email: tfuchigami@ims.gr.jp

doi: https://doi.org/10.14740/ijcp460 static intolerance with recurrent syncope and orthostatic dizziness. These abnormalities are known as orthostatic dysregulation (OD). OD is a clinical entity, and children with OD have various symptoms, such as recurrent dizziness, chronic fatigue, headache, and syncope, resulting in impairment of quality of life in proportion to the severity of the illness [1,2]. Previous Japanese studies reported that the prevalence of OD increases before adolescence and reaches a peak of $15 \%$ in adolescents aged 14 - 15 years [3].

Cerebrospinal fluid (CSF) hypovolemia was first reported as "aliquorrhea" in 1938 [4]. Since the 1990s, CSF hypovolemia in adults has been recognized using diffuse pachymeningeal gadolinium enhancement on magnetic resonance imaging (MRI) [5].

In Japan, CSF hypovolemia was first reported in a case of CSF leakage due to mild traffic trauma in 2003 [6]. The first comprehensive report of CSF hypovolemia in children was presented at the 13th International Headache Congress in 2007 [7], and reports on children have been scattered since then $[8,9]$.

The essential mechanism of this disease is thought to be the CSF leakage at the spinal level, which decreases the volume and pressure of the CSF and induces a broad spectrum of clinical manifestations [10].

The causes of CSF hypovolemia in children remain unclear, and patients with this condition complain of symptoms, such as orthostatic headache, vertigo, and tinnitus; therefore, it can be represented by OD.

Herein, we present a case of a pediatric patient with CSF hypovolemia followed by OD.

\section{Case Report}

A previously healthy 13 -year-old boy presented with orthostatic headache, vertigo, and vomiting for 2 weeks. He enjoyed his daily life playing soccer until those symptoms appeared. $\mathrm{He}$ visited the pediatric clinic and received an intravenous infusion, which temporarily improved his symptoms, but the headaches and dizziness recurred. He then visited an otolaryngologist the next day; however, on physical examination, no abnormalities were found. Furthermore, he underwent a head computed tomography (CT) examination, but no abnormal findings were found. He was able to rise normally in the morning and participated in soccer training, but he visited our hospital when there was no improvement in his headache and vertigo. He had no remarkable medical, family, psychosocial, 
Table 1. Criteria of Orthostatic Dysregulation

Major manifestations

A. Susceptibility to vertigo and dizziness on standing

B. A tendency for fainting in the standing position, which in severe cases leads to a fall

C. Nausea on taking a hot bath or on encountering unpleasant experiences

D. Palpitation and/or dyspnea after mild exercise

E. Difficulty in getting out of bed

Minor manifestations

a. Pallor

b. Anorexia

c. Occasional umbilical colic (severe abdominal pain)

d. Fatigability

e. Frequent headache

f. Motion sickness

g. Decrease in pulse pressure of $>16 \mathrm{~mm} \mathrm{Hg}$ during standing in the postural test

h. Decrease in systolic blood pressure $>21 \mathrm{~mm} \mathrm{Hg}$ during standing in the postural test

i. Increase in pulse rate of $>21 / \mathrm{min}$ during standing in the postural test

j. Decrease in TI, TII amplitude on electrocardiogram of $>0.2 \mathrm{mV}$ during standing in the postural test

The presence of either combined one major and three minor, combined two major and one minor, or three major manifestations indicates a high probability of orthostatic dysregulation.

birth, developmental, or vaccination histories.

At the time of the visit to our hospital, he was $157(-0.79$ standard deviation (SD)) $\mathrm{cm}$ in height and weighed $37(-1.35$ SD) $\mathrm{kg}$. His body temperature was $37.0^{\circ} \mathrm{C}$, pulse rate was 48 beats/min, and blood pressure was 105/52 mm Hg. He was conscious and aware of his surroundings, but he was quiet and not normally active for a child his age. No neurological abnormalities were observed. Brain MRI revealed no specific findings. Blood examination revealed mild iron deficiency anemia (hemoglobin level, $10.0 \mathrm{~g} / \mathrm{dL}$; hematocrit, 32.1\%; serum iron, $16 \mu \mathrm{g} / \mathrm{dL}$; total iron-binding capacity, $513 \mu \mathrm{g} / \mathrm{dL}$; serum ferritin, $5.0 \mathrm{ng} / \mathrm{mL}$ ), and oral administration of sodium ferrous citrate was started. However, after 3 months, the patient's headache worsened, and then the orthostatic test (Table 1) [13] was performed, leading to a working diagnosis of OD. In the postural test, systolic and diastolic blood pressures, pulse rate, and electrocardiogram were measured after $10 \mathrm{~min}$ in the supine position and after $10 \mathrm{~min}$ of active standing. The postural test showed a significant increase in pulse rate (48 beats/ min in the supine position, 75 beats/min during standing) and a significant decrease in pulse pressure $(53 \mathrm{~mm} \mathrm{Hg}$ in the supine position, $32 \mathrm{~mm} \mathrm{Hg}$ during standing), while his blood pressure was $105 / 52 \mathrm{~mm} \mathrm{Hg}$ in the supine position and $104 / 72 \mathrm{~mm} \mathrm{Hg}$ during standing. Two major symptoms (Table 1, A and D) and five minor symptoms (Table 1, b, e, g, i, and j) of the diagnostic criteria for OD were met. He was diagnosed with OD and began oral administration of midodrine hydrochloride, $2 \mathrm{mg}$ tablets twice daily (in the morning and evening); after taking the medication for 4 months, some symptoms improved, but the headache worsened. Psychological counseling was proposed. However, he had no trouble meeting the demands of school life and showed no signs of psychological concerns; therefore, his psychological intervention was postponed. Subsequently, he began to take a break from school because his palpitation appeared during soccer practice, and his fatigue and headache worsened after returning home from school. His diagnosis of OD was questioned because therapy with oral administration of midodrine hydrochloride was ineffective, and his symptoms were exacerbated from afternoon to night, not morning. These findings indicated CSF hypovolemia as the diagnosis. He underwent an examination at the Sanno Hospital. Radioisotope (RI) cisternography was performed by intrathecal lumbar injection of $1 \mathrm{~mL}$ (37 MBq at calibration time) of ${ }^{111}$ In (Nihon Medi-Physics, Japan) under local anesthesia with 3 - $5 \mathrm{~mL}$ of $1 \%$ lidocaine, with the patient in the lateral decubitus position. Posteroanterior and anteroposterior whole-body planar scintigraphy was performed using a single-headed gamma camera (RC 2500 IV; Hitachi Medical, Tokyo, Japan) at 1, 3, 5, and $24 \mathrm{~h}$ after injection. RI cisternography images after RI injection showed no apparent parathecal activity, except early bladder filling at $3 \mathrm{~h}$ (normal: RI bladder filling after $5 \mathrm{~h}$ ), slight parathecal activity at the lumbar level at $5 \mathrm{~h}$, and decreased RI residual activity of $20.7 \%$ at $24 \mathrm{~h}(\leq 30 \%$ at $24 \mathrm{~h}$ after RI injection, Fig. 1). CT myelography showed mild bilateral extrathecal CSF collections at the lumbar 2 - 3 level (Fig. 2). Lumbar CSF pressure was also measured at the time of intrathecal injection of RI via lumbar puncture in the lateral position. The patient's CSF pressure was normal $\left(13 \mathrm{~cm} \mathrm{H}_{2} \mathrm{O}\right)$. However, RI cisternography revealed CSF leaks mainly in the lumbar spine, early bladder filling, and accelerated RI clearance rate, and CT myelography confirmed the location of the CSF leakage. According to the diagnostic criteria of the international classification of headache disorders, third edition [11], the patient was diagnosed with CSF hypovolemia. Epidural blood 


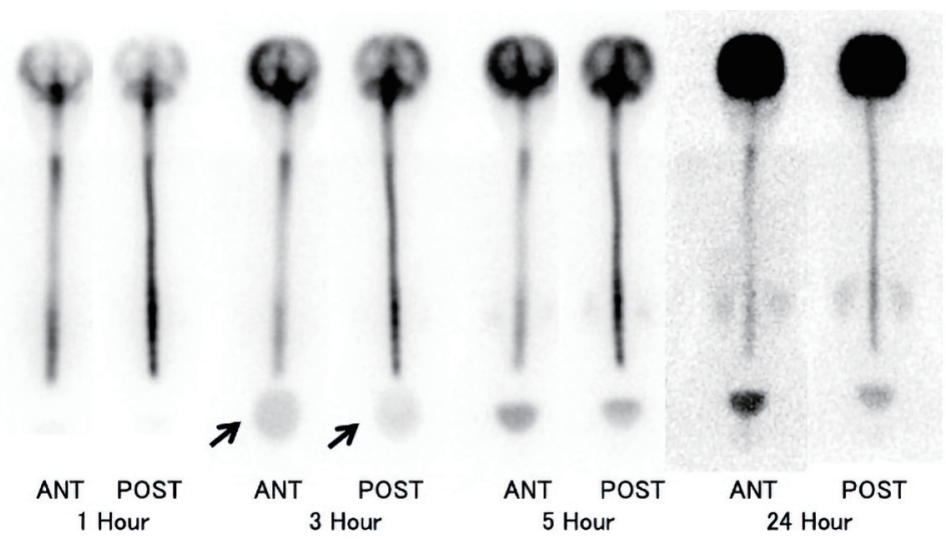

Figure 1. Radioisotope (RI) cisternography images. RI cisternography images at $1,3,5$, and $24 \mathrm{~h}$ after RI injection. Early bladder filling at $3 \mathrm{~h}$ (arrow) and decreased RI residual activity $(20.7 \%)$ at $24 \mathrm{~h}$ were observed. ANT: anterior view; POST: posterior view.

patch (EBP) treatment was performed $(21 \mathrm{~mL}$ of autologous blood was injected into the epidural space at the lumbar 2 - 3 level in the prone position). After this treatment, his headache improved. At an early stage after treatment, he needed an oral administration of midodrine hydrochloride, $2 \mathrm{mg}$ tablets once or twice daily for 6 months. However, with the increase in daily activity, no symptoms were observed even without the medication, and he is now able to participate in school activities without symptoms 5 years after EBP treatment.

\section{Discussion}

Patients with both CSF hypovolemia and OD present with orthostatic headache [12]. Moreover, CSF hypovolemia and OD

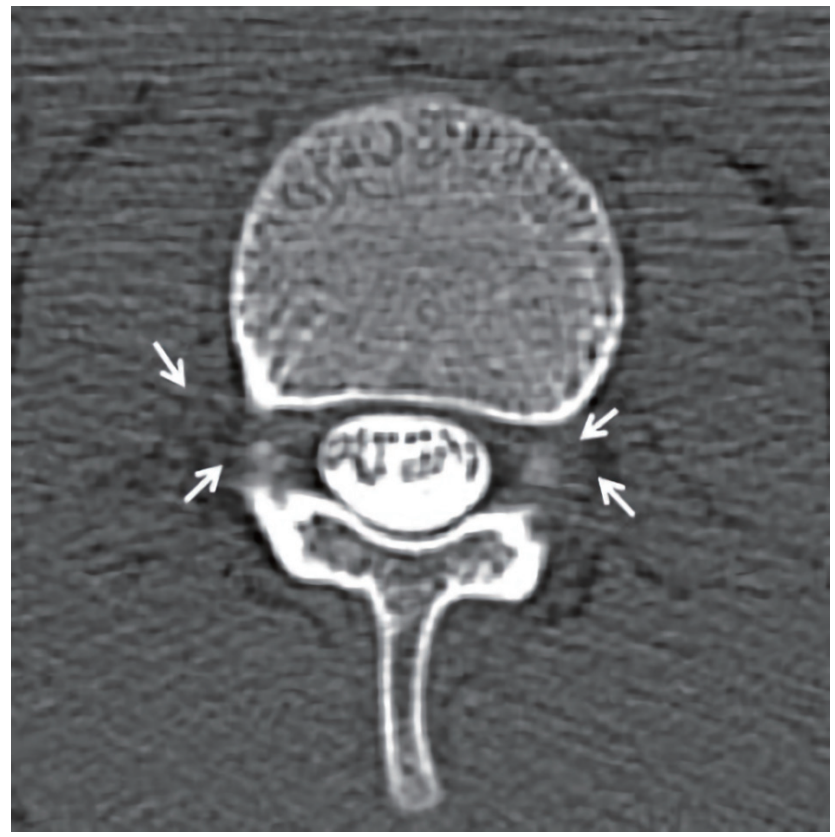

Figure 2. Postmyelography contrast computed tomography (CT). Axial CT showing bilateral extrathecal cerebrospinal fluid collections (arrows). have similar symptoms. Therefore, some patients with CSF hypovolemia are misdiagnosed with OD, autonomic nervous system dysfunction, or mental disorders. In our case, the patient was also misdiagnosed with OD.

Patients with CSF hypovolemia usually require rest in the early stage of symptoms onset because the symptoms tend to worsen with exercise and activity; however, patients with OD can tolerate high level of activity in the early stage of symptoms onset. The initial responses to therapy in each condition are different; therefore, these findings are important for distinguishing between the two conditions. Orthostatic headache caused by CSF hypovolemia usually occurs gradually after a standing load and is accompanied by photosensitivity, tinnitus, and a feeling of ear obstruction. It is characterized by an exacerbation of symptoms from afternoon to night (Table 2) [13]. Although it has been reported that such typical orthostatic headaches are rarely exhibited [14], the onset time and degree of headache vary, and even the same child may present different daily situations. Our patient was able to go to school and participate in soccer club activities normally, but after returning home, his headache and fatigue appeared, and he needed to be in a supine position. These symptoms were clearly different from that of OD, but it was difficult and time-consuming to diagnose as an orthostatic headache due to CSF hypovolemia. In addition, this patient visited three clinics and one hospital within the first 2 days of the onset, indicating that the symptom onset was sudden. In contrast, the symptoms of OD are insidious in onset instead of sudden.

The most common cause of CSF hypovolemia is trauma, such as from sports, physical exercise at school, collisions with other children, falls, and traffic accidents while biking or walking. However, more than half of the causes are unknown and are not rare. In the present case, the patient was active in soccer, but the cause of CSF hypovolemia was still unknown. CSF in children is more likely to be injured and leak than in adults because their arachnoid and dura are underdeveloped and fragile, and children are active and have higher tendencies to participate in sporting activities [15].

Initially, the main method of diagnosing CSF hypovolemia was RI cisternography; however, with the progress of diagnos- 
Table 2. Differentiation of Orthostatic Headache Between Cerebrospinal Fluid (CSF) Hypovolemia and Orthostatic Dysregulation (OD)

\begin{tabular}{lll}
\hline & CSF hypovolemia & OD \\
\hline Time to onset of headache & Gradually after standing load & Immediately after standing load \\
Photosensitivity & Positive & Negative \\
Tinnitus/feeling of ear obstruction & Positive & Negative \\
Analgesics & No effect & No effect \\
Exercise & Exacerbation & Light \\
Diurnal variation of symptoms & Exacerbation from afternoon to night & Light from afternoon to night \\
\hline
\end{tabular}

tic imaging, the combined use of RI cisternography and CT myelography has been recognized, and currently, spinal cord MRI has become the first-line diagnostic method. Definitive diagnosis requires RI cisternography and MRI/CT myelography; however, because of the need for lumbar puncture, problems, such as radiation exposure, and the fact that typical imaging findings may not always be obtained, it is often difficult to diagnose CSF hypovolemia. In recent years, reports on the usefulness of spinal cord MRI have been increasing [14, 16, 17]; however, spinal cord MRI was not performed for our patient because it was not yet a routine examination in children. If minimally invasive MRI can be used for diagnosis, it will lead to the diagnosis and treatment of more children.

In the present case, the patient's CSF pressure was normal. Schievink et al reported that among the 19 patients with CSF hypovolemia, $11(58 \%)$ had CSF pressure below normal $(<6.5$ $\left.\mathrm{cm} \mathrm{H}_{2} \mathrm{O}\right)$, and eight $(42 \%)$ had CSF pressure within the normal range [15], indicating that CSF pressure in patients with CSF hypovolemia is not always low.

In children, CSF hypovolemia may be improved by conservative treatments, such as rest, intravenous infusion, and sufficient fluid intake. In our case, when the conservative treatments became ineffective, EBP was performed; autologous blood was injected into the epidural space around the CSF leakage site according to the RI cisternography. If the CSF leakage was unclear, autologous blood was injected at the lumbar 2 - 3 level in a prone position under local anesthesia. The outcomes of EBP treatment are reported to be better in children than in adult cases. After EBP treatment, 95.9\% of pediatric and adolescent patients with CSF hypovolemia showed good or moderate improvement [9].

In CSF hypovolemia, in addition to headache and malaise, various symptoms including visual, auditory, and higher cerebral dysfunctions, urinary incontinence, autonomic dysfunction, such as thermoregulation disorder and abnormal sweating, can be observed [18]. The mechanism of these symptoms caused by CSF leakage has not yet been fully elucidated. The decrease in CSF is thought to cause the brain to hang down, pulling blood vessels, nervous tissue, and dura mater, leading to headaches and cranial nerve palsy [19].

It is important to note that CSF hypovolemia is not rare in children, and that it may be found among children with OD, chronic daily headaches, and indeterminate complaints, which interfere with school and daily activities. However, in reality, it seems that the number of cases leading to the diagnosis of CSF hypovolemia in children is limited, and it is necessary to consider the possibility that it has been misdiagnosed. If the symptoms are severe and the patient cannot go to school, the patient may be diagnosed with psychiatric disorders, such as somatic symptom disorder and depression [20]. Increasing indeterminate complaints as the symptoms persist make the diagnosis even more difficult. In long-term cases, it is important to carefully observe the symptoms at the onset, especially within 2 weeks [21].

\section{Learning points}

It is important to note that CSF hypovolemia is not rare in children and that it may be found among children with OD, chronic daily headaches, and indeterminate complaints, which interfere with school and daily activities.

\section{Acknowledgments}

None to declare.

\section{Financial Disclosure}

The authors received no financial support for the research and/ or authorship of this article.

\section{Conflict of Interest}

The authors declare no potential conflict of interest with respect to the authorship and/or publication of this article.

\section{Informed Consent}

Informed written consent for publication of this case was obtained from the patients' parents.

\section{Author Contributions}

Y. Ohashi, S. Takahashi, Y. Moriuchi, T. Kamioka, T. Fuchiga- 
mi, and K. Takahashi provided medical care and contributed to the preparation of the manuscript. Y. Ohashi and T. Fuchigami drafted the manuscript. T. Fuchigami designed the manuscript outline and provided conceptual advice. K. Takahashi supervised the study. All authors read and approved the final manuscript.

\section{Data Availability}

The authors declare that data supporting the findings of this study are available within the article.

\section{References}

1. Okuni M. Orthostatic dysregulation in childhood with special reference to the standing electrocardiogram. Jpn Circ J. 1963;27:200-204.

2. Tanaka H, Fujita Y, Takenaka Y, Kajiwara S, Masutani S, Ishizaki Y, Matsushima R, et al. Japanese clinical guidelines for juvenile orthostatic dysregulation version 1 . Pediatr Int. 2009;51(1):169-179.

3. Honda K, Nose T, Yoshida N, Tanimura M, Tanaka K. Responses to the postural change and orthostatic dysregulation symptoms: a population study on Japanese junior and senior high school students. Jpn Circ J. 1977;41(6):629641.

4. Schaltenbrand G. Neuere Anschauungenzur Pathophisiologie der Liquorzirkulation. Zentralbl Neurochir. 1938;3:290-300.

5. Mokri B, Krueger BR, Miller GM, Piepgras DG. Meningeal gadolinium enhancement in low-pressure headaches. J Neuroimaging. 1993;3(1):11-15.

6. Shinonaga M, Suzuki S. Diagnosis and treatment of intracranial hypotension (cerebrospinal fluid hypovolemia). Neurotraumatology (in Japanese). 2003;26(2):98-102.

7. Shinonaga M. Cerebrospinal fluid hypovolemica (intracranial hypotension) in children and adolescents. Cephalalgia. 2007;27:632.

8. Takahashi K, Mima T. Cerebrospinal fluid hypovolemia in childhood and adolescence: clinical feature and outcomes. Childs Nerv Syst. 2011;36(6):552-559.

9. Takahashi K. Cerebrospinal fluid leakage in childhood and adolescence: analysis of 195 patients and literature review. Childs Nerv Syst. 2019;44(4):317-323.

10. Mokri B. Spontaneous cerebrospinal fluid leaks: from intracranial hypotension to cerebrospinal fluid hypovolemia - evolution of a concept. Mayo Clin Proc. 1999;74(11):1113-1123.

11. Headache Classification Committee of the International Headache Society (IHS). The international classification of headache disorders, 3rd edition. Cephalalgia. 2018;38(1):1-211.

12. Mokri B, Low PA. Orthostatic headaches without CSF leak in postural tachycardia syndrome. Neurology. 2003;61(7):980-982.

13. Hashimoto Y, Wada K. Diagnosis and treatment of cerebrospinal fluid hypovolemia. Neurology (in Japanese). 2020;92(4):385-391.

14. Moriyama E, Ishikawa S, Fujiwara M. Current management for pediatric patients with spinal cerebral spinal fluid leak. Childs Nerv Syst (in Japanese). 2020;45(4):332339.

15. Schievink WI, Maya MM, Louy C, Moser FG, Sloninsky L. Spontaneous intracranial hypotension in childhood and adolescence. J Pediatr. 2013;163(2):504-510.

16. Osawa I, Kozawa E, Mitsufuji T, Yamamoto T, Araki N, Inoue K, Niitsu M. Intravenous enhanced 3D FLAIR imaging to identify CSF leaks in spontaneous intracranial hypotension: Comparison with MR myelography. Eur J Radiol Open. 2021;8:100352.

17. Hosoya T, Hatazawa J, Sato S, Kanoto M, Fukao A, Kayama T. Floating dural sac sign is a sensitive magnetic resonance imaging finding of spinal cerebrospinal fluid leakage. Neurol Med Chir (Tokyo). 2013;53(4):207-212.

18. Vilming ST, Mokri B. Low cerebrospinal fluid pressure. In: Olsen J, Goadsby PJ, et al., eds. The Headache. 3rd ed. Philadelphia: Lippincott Williams \& Wilkins, 2006. p. 935-944.

19. Levine DN, Rapalino O. The pathophysiology of lumbar puncture headache. J Neurol Sci. 2001;192(1-2):1-8.

20. Shinonaga M. Relations between cerebrospinal fluid hypovolemia and psychosomatic disorder. Jpn J Psychosom Med. (in Japanese). 2014;54(11):1026-1033.

21. Mitsufuji T, Araki N. Orthostatic disturbance. Jpn J Med Pharm Sci (in Japanese). 2016;73:1383-1386. 